\title{
Europe hooks up with China for space first
}

\section{Sally Goodman, Paris}

European space experiments will fly on board Chinese satellites for the first time in a joint project to study the magnetosphere - the magnetic shield that surrounds the Earth.

The mission, known as Double Star, was announced on 9 July by the European Space Agency (ESA) and the Chinese National Space Administration. It will involve two small Chinese satellites flying in orthogonal orbits - around the poles and the equator, respectively (see right) - to gather data on how the Sun affects the magnetosphere's behaviour.

In its first substantial collaboration in space science with China, the ESA will contribute 10 of the project's 18 experiments, costing about 8 million euros (US\$6.8 million). ESA officials are confident that the project will lead to more extensive collaborations with China in the future - despite American misgivings about sharing space technology with China.

Double Star is the latest of several projects designed to investigate the relationship between the Sun and the magnetosphere. Interaction between solar winds and the magnetosphere cause spectacular physical effects, including the aurora - or northern lights - seen over the polar skies, and massive magnetic storms that disrupt electricity supplies and radio communications.

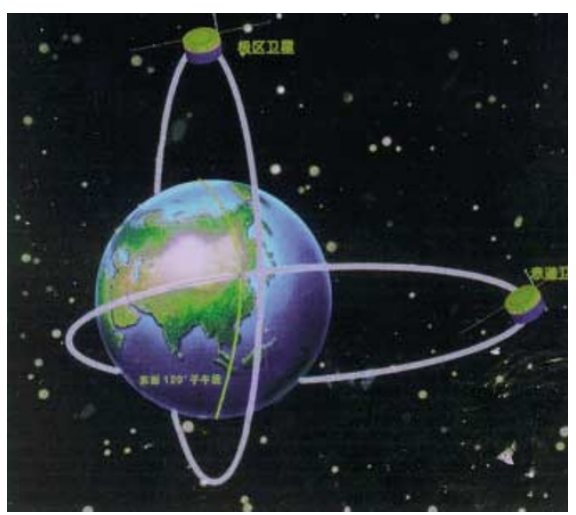

Doubleplusgood: the orbits of the two satellites will allow investigation of the magnetosphere.

But many of these projects have suffered from technical glitches. Germany's EquatorS satellite, for example, was launched in 1997 but stopped sending back data after only five months when its batteries failed. And Cluster, a project involving the ESA, NASA and other partners, was lost in 1996 when its Ariane 5 rocket launcher failed.

But Cluster - a flotilla of four identical spacecraft - was relaunched last summer, and Michael Fehringer, an ESA scientist working on the project, says early data indicate that the mission "will revolutionize our understanding of the magnetosphere".

The ESA instruments that will fly on
Double Star are identical to those on Cluster, but they will fly in different orbits. To keep costs down, the equipment will mostly be put together using spare parts left over from Cluster. But because of US restrictions on exports to China, instruments of American origin will be rebuilt in Europe for the new mission. For example, André Balogh of Imperial College, London, says that his Double Star experiment will use a Britishbuilt magnetometer to replace the American one he used on Cluster.

The ESA hopes that the Double Star satellites, which are scheduled for launch on Chinese Long March 2C rockets in December 2002 and March 2003, will operate in parallel with the four existing Cluster spacecraft. Cluster is only funded until January 2003, but the Europeans hope to extend its life by at least two years.

Even if this does not happen, Double Star will allow researchers to gather new information, says Andrew Fazakerley of the Mullard Space Science Laboratory at University College London, who is leading one of the experiments. A process called magnetic reconnection - whereby particles are accelerated towards Earth's magnetic poles from its huge magnetic tail - will be observed in unprecedented detail from Double Star's equatorial orbit, he says.

http://www.esa.int

\section{Institutes prepare for pioneering bioinformatics work}

\section{Declan Butler, Paris}

Two women scientists are to take control of two of Europe's leading bioinformatics initiatives, with a remit to expand intramural research activities.

Janet Thornton, a structural-biology professor at University College London, has been named as research director of the European Bioinformatics Institute (EBI) near Cambridge in Britain. She succeeds Michael Ashburner, who has co-directed the centre with Graham Cameron since it opened five years ago.

And Nadia Rosenthal, an associate molecular-biology professor at Harvard University, will succeed Klaus Rajewsky as head of the mouse biology programme at the European Mouse Mutant Archive near Rome.

Both facilities are affiliated to the European Laboratory of Molecular Biology (EMBL), and have until now acted chiefly as service providers to outside researchers. But Fotis Kafatos, director general of the EMBL, says that the laboratory is now shifting its emphasis to functional genomics, and he expects the facilities to play a more active role

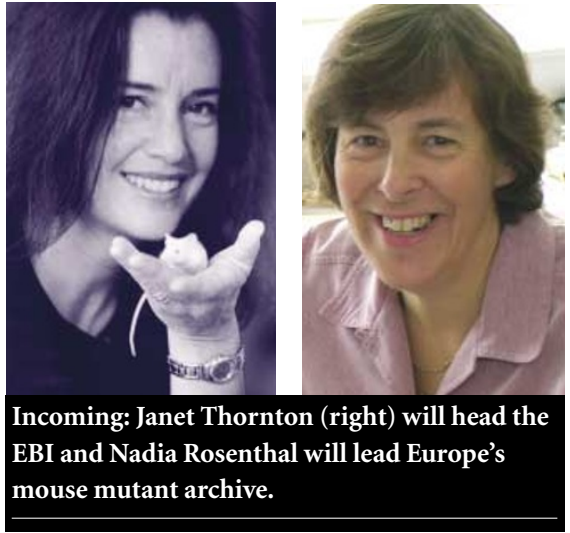

in pioneering original research of their own.

The EBI recently received a multimillion grant from the European Union (see Nature 411,229 ; 2001), securing its main database programmes. Thornton says that the next step is to build a research environment around these projects. "To make progress in understanding whole systems we need to integrate these genomic, proteomic and other resources," she says.

Thornton's main research interests are in the analysis and modelling of protein structures and in rational drug design. She says that she is keen to add 'chemoinformatics' to the EBI's traditional bioinformatics role and to strengthen the links between the EBI and the pharmaceutical industry.

Ashburner, who has split his time between the EBI and his faculty position at the University of Cambridge, will now return to full-time research at the university. But he says that he is keen to help Thornton settle in, and will remain involved with the EBI to oversee a large expansion of its gene ontology (GO) consortium, an effort to build controlled vocabularies that will assist in the cross-searching of biological databases (see Nature 411, 631-632; 2001).

Approaches such as GO are absolutely critical, says Thornton. "Without being able to describe function in a computer-readable form - without functional ontologies - we will not be able to describe whole systems." http://www.ebi.ac.uk http://www.emma.rm.cnr.it

i http://www.geneontology.org 